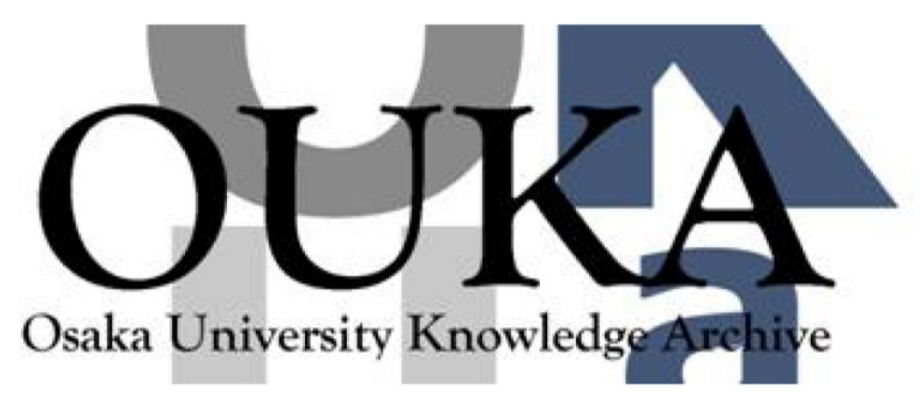

\begin{tabular}{|c|c|}
\hline Title & $\begin{array}{l}\text { Observation of neutron spectrum produced by } \\
\text { fast deuterons via ultraintense laser plasma } \\
\text { interactions }\end{array}$ \\
\hline Author (s) & Izumi, N.; Sentoku, Y.; Habara, H. et al. \\
\hline Citation & Physical Review E. 65(3) p.036413 \\
\hline Issue Date & $2002-03$ \\
\hline oaire:version & VoR \\
\hline URL & https://hdl. handle. net/11094/3023 \\
\hline rights & $\begin{array}{l}\text { Izumi, N., Sentoku, Y., Habara, H., Takahashi, } \\
\text { K., Ohtani, F., Sonomoto, T., Kodama, R.. } \\
\text { Norimatsu, T.,'Fujita, H., Kitagawa, Y., Mima, } \\
\text { K. 'Tanaka, K. A., Yamanaka, ,. Physical Review } \\
\text { E, 65, 3, 036413, 2002-03. "Copyright } 2002 \text { by } \\
\text { the American Physical Society." }\end{array}$ \\
\hline Note & \\
\hline
\end{tabular}

Osaka University Knowledge Archive : OUKA

https://ir. Library. osaka-u. ac. jp/

Osaka University 


\title{
Observation of neutron spectrum produced by fast deuterons via ultraintense laser plasma interactions
}

\author{
N. Izumi, ${ }^{1, *}$ Y. Sentoku, ${ }^{1}$ H. Habara, ${ }^{1, \dagger}$ K. Takahashi, ${ }^{1}$ F. Ohtani, ${ }^{1}$ T. Sonomoto, ${ }^{1}$ R. Kodama, ${ }^{1}$ T. Norimatsu, ${ }^{1}$ H. Fujita, ${ }^{1}$ \\ Y. Kitagawa, ${ }^{1}$ K. Mima, ${ }^{1}$ K. A. Tanaka, ${ }^{1,2}$ and T. Yamanaka ${ }^{1}$ \\ ${ }^{1}$ Institute of Laser Engineering, Osaka University, Suita, Osaka 565-0871, Japan \\ ${ }^{2}$ Department of Electro, Information System, and Energy Engineering, Osaka University, Suita 565-0871, Japan
}

(Received 9 August 2001; published 6 March 2002)

\begin{abstract}
We report the first precise spectral measurement of fast neutrons produced in a deuterated plastic target irradiated by an ultraintense sub-picosecond laser pulse. The 500-fs, 50-J, 1054-nm laser pulse was focused on the deuterated polystyrene target with an intensity of $2 \times 10^{19} \mathrm{~W} / \mathrm{cm}^{2}$. The neutron spectra were observed at $55^{\circ}$ and $90^{\circ}$ to the rear target normal. The neutron emission was $7 \times 10^{4}$ per steradian for each detector. The observed neutron spectra prove the acceleration of deuterons and neutron production by $d(d, n)^{3} \mathrm{He}$ reactions in the target. The neutron spectra were compared with Monte Carlo simulation results and the deuteron's directional anisotropy and energy spectrum were studied. We conclude that $2 \%$ of the laser energy was converted to deuterons, which has an energy range of $30 \mathrm{keV}$ up to $3 \mathrm{MeV}$.
\end{abstract}

DOI: 10.1103/PhysRevE.65.036413

\section{INTRODUCTION}

Recent development of a chirped-pulse-amplification (CPA) technique has made it possible to focus a laser pulse with irradiation intensity over $10^{19} \mathrm{~W} / \mathrm{cm}^{2}$ [1]. In this region, the radiation pressure of a $1-\mu \mathrm{m}$ laser exceeds 3 Gbar and the laser fields oscillate electrons in relativistic velocities. The production of multi-MeV electrons in ultraintense lasermatter interaction is reported [2]. The most exciting application of these energetic electrons is a "fast ignitor scheme" proposed as a new concept of inertial fusion energy [3]. The first ignitor scheme anticipates an additional heating of imploded fuel plasma by using suprathemal electrons produced through ultraintense laser-plasma interactions. This new scheme has intensified interest in the high-intensity lasermatter interactions. On the other hand, the production of energetic ions in ultraintense laser-matter interaction was also observed [4-10]. The energy deposition of the energetic ions could influence the core dynamics of the fast ignition [4]. If the efficiency of the energetic ion production is sufficient, there might be a possibility of an additional core heating via fast ions. To evaluate the influence of energetic ions on the implosion dynamics, it is crucial to study the energy transfer to ions.

We observed energetic ion spectra from ultraintense lasermatter interactions with an indirect technique using neutron spectroscopy. Previously, energetic ions were observed directly with charged particle detectors [5]. In some cases, the energies and charge states of ions were resolved via electric and magnetic fields. The energy could be also resolved by difference of exposure of layered film detectors (stack of CR39s, radiochromic films, and/or Ti activation foils). The

\footnotetext{
*Corresponding author. Present address: Lawrence Livermore National Laboratory, P.O. Box 8087000 East Avenue L-399 Livermore, CA 94550. Email address: izumi2@1lnl.gov

${ }^{\dagger}$ Present address: Rutherford Appleton Laboratory Chilton, Didcot, Oxon OX11 0QX, UK.
}

PACS number(s): 52.38.Kd, 52.57.Kk, 52.59.-f, 52.50.Jm angular distribution of the energetic ions was observed as exposure images of these films. The advantages of the direct observation of charged particles are (1) high sensitivity and large dynamic range of detectors, (2) easy access to the energy resolution with range filters or a magnetic field, and (3) easy access to imaging by radiation sensitive films. However, since the detector observes the ions from outside the target, the energies and directionality of ions can be influenced by (1) energy slowdown inside the target and (2) deflection by the electrostatic or magnetic fields around the target. The charged particle observation is sensitive not only to the ions accelerated at the irradiated surface but also to that accelerated at the rear surface [5].

The observation of nuclear reactions inside the target is an alternative method of energetic ion measurement [6-9]. When a deuterated plastic target was used, energetic deuterons caused $d(d, n)^{3} \mathrm{He}$ reactions with deuterons in the target. The energy spectrum of neutrons contains the information on the energetic deuterons. Since the target itself was used as the ion-neutron converter, this method is sensitive only to the ions accelerated into the target. The sensitivity and dynamic range of this method is governed by the cross section of the nuclear reactions and the dynamic range of the neutron detectors. However, due to their large mean free path, the neutrons directly bring out the information on energetic ions from the interacting region. Especially for the ions between $30 \mathrm{keV}$ and a few $\mathrm{MeV}$, the nuclear reaction method is the only way to observe these ions, since they are stopped in the target.

The first neutron spectrum in high-intensity laser irradiation was reported by Norreys et al. [7]. They observed the neutron spectrum by an etch track diameter analysis of CR39 using the maximum entropy method and current mode scintillation detectors with few-meter flight paths. Because of the accuracy of their spectral measurement, discussion of the of neutron production mechanism was postponed to a future study. Recently, the angular distribution of fast neutron production inside the target was observed with a $\mathrm{BF}_{3}$ tube counter [8]. The coarse neutron spectrum that was observed by 96 -channel single hit mode time-of-flight detector array 


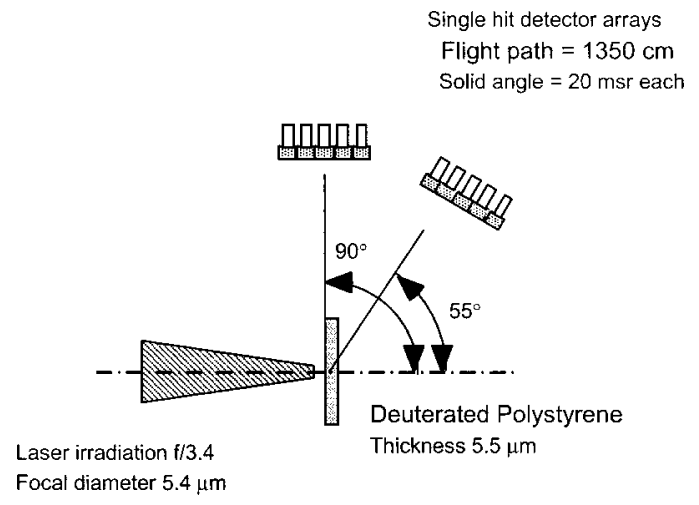

FIG. 1. A schematic view of the experimental setup. The neutron time-of-flight detector arrays were located $13.5 \mathrm{~m}$ away from the target with angles of $55^{\circ}$ and $90^{\circ}$ to the rear target normal.

and qualitative analysis of the spectrum are also reported by Disdier, Garçonnet, and Miquel [9]. The precise neutron energy spectrum is of crucial importance to a study of the mechanism of both ion acceleration and neutron production.

Previously, we reported preliminary results and qualitative interpretation of the spectrum of neutrons produced in the deuterated plastic target irradiated by a 500 -fs laser pulse at an intensity of $2 \times 10^{19} \mathrm{~W} / \mathrm{cm}^{2}$ [10]. Here we report the details of the experiment and the quantitative analysis of the neutron spectrum. We have clarified that the observed neutrons are produced by $d(d, n)^{3} \mathrm{He}$ reactions in the target. The ion accelerated on an irradiated surface has been clearly separated from that on a rear side surface. The directionality and energy spectrum of energetic deuterons also have been revealed by a spectrum unfolding technique using Monte Carlo simulation. We have compared the experimental results with the particle in cell simulations and mechanisms of ion acceleration are discussed.

\section{DESCRIPTION OF EXPERIMENT}

Figure 1 shows the schematic of the experiment. The target was a deuterated polystyrene $\left(\mathrm{C}_{8} \mathrm{D}_{8}\right)_{x}$ film of $5.5 \mu \mathrm{m}$ thickness. This target was irradiated by CPA Nd glass laser "Peta Watt module" (PWM) [11]. The PWM laser was operated at $\lambda=1054 \mathrm{~nm}$. The output energy was $50 \mathrm{~J}$ and the pulse width was $500 \mathrm{fs}$ full width at half maximum. The laser pulse was directed at the target chamber and focused by an on-axis parabolic mirror. The target was irradiated from the target normal with the focal diameter of $5.4 \mu \mathrm{m}$ full width at half maximum. The intensity of the focal spot was 2 $\times 10^{19} \mathrm{~W} / \mathrm{cm}^{2}$. At $0.8 \mathrm{~ns}$ before the main pulse, there was a prepulse, which had a few times $10^{-4}$ of main pulse energy.

The energy spectrum of fast electrons produced by highintensity laser pulse irradiation was observed by an electron spectrometer at an angle of $42^{\circ}$ to the laser propagation direction The spectrometer uses a pair of magnet plates with a $1.2 \mathrm{kG}$ magnetic strength. Since it is insensitive to the background due to a strong burst of electromagnetic pulse, an imaging plate was used as a detector [10].

We measured the neutron spectrum by using time-of-flight detector arrays "MANDALA" [12]. MANDALA has two sets of single-hit scintillation detector arrays (421 channels $\times 2$ set). Each array is located $13.5 \mathrm{~m}$ away from the target with an angle of $55^{\circ}$ and $90^{\circ}$ to the direction of the CPA laser propagation. Each detector array covers a solid angle of 20 $\mathrm{msr}$. When a neutron recoils a proton in a plastic scintillator, the recoiled proton causes scintillation. The scintillation light is detected by a photomultiplier. The signal pulse is guided to discriminators and only those above the threshold are picked. The discriminator threshold of $50 \mathrm{mV}$ corresponds to the recoiled proton energy of $1.3 \mathrm{MeV}$. An electric trigger pulse, which is synchronized to the laser pulse, starts time-to-digital converters, which records the detection timing of each neutron signal. The detection efficiency is calibrated absolutely using an accelerator neutron source. The energy of each neutron is resolved by the time-of-flight method. The energy resolution is $30 \mathrm{keV}$ for $2.5 \mathrm{MeV}$ neutrons.

In the ultraintense laser experiment, excessive amounts of high-energy electrons are generated and these energetic electrons cause an intense bremsstrahlung flash in the target, on the instruments close to the targets, and on the irradiation chamber walls. This intensive bremsstrahlung flash hits the detector and prevents subsequent neutron detection during detector's dead time. To reduce this undesirable interference, cone-shaped "snout" collimators are installed in the irradiation chamber. The snout collimators limit the view of the detectors and reduce the bremsstrahlung flash from the diagonal inner walls of the irradiation chamber. The bodies of collimators were made of $1-\mathrm{cm}$-thick lead cylinders. To reduce bremsstrahlung on the collimator itself, the tops of the lead collimators were covered with 4.5 -cm-thick low- $Z$ (plastic) electron moderators. The neutron detectors observe the target through a hole bored through the center of each electron moderator. Therefore, these low- $Z$ electron moderators also work as neutron collimators. The thick walls of the irradiation chamber (8-cm-thick stainless steel) also contribute to reducing the photon flash leaking out of the chamber. We placed a current mode scintillation detector also in the forward direction $\left(0^{\circ}\right.$ to the laser propagation direction). However, the detector was saturated by a strong burst of bremsstrahlung and we could not observe a neutron signal at $0^{\circ}$.

Once an element of MANDALA detects a neutron signal, the element cannot detect subsequent neutrons during discriminator dead time (100 ns). When a fraction of the channels are in their dead time, the number of live channels (i.e., the total detection efficiency) is decreased. This temporal fluctuation of active channel number is also recorded and the distortion of the neutron spectrum due to this effect is compensated for.

Figure 2 shows typical neutron spectra observed in this experiment. The upper graph shows the spectrum observed at $55^{\circ}$ and the lower at $90^{\circ}$. The solid circle shows the experimental results. The error bar means $68 \%$ confidence interval of the statistical error of each energy bin. The vertical axes of these spectra are compensated by taking into account detection efficiencies for each energy bin. The solid and dashed lines are the ones obtained by numerical fitting mentioned in a later section.

These spectral shapes were reproducible during several 


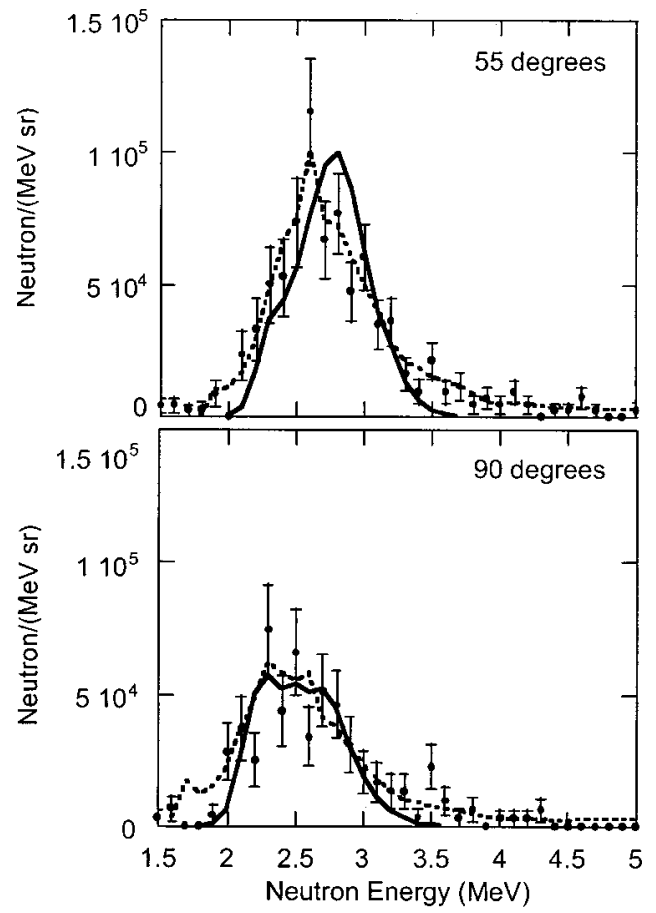

FIG. 2. The neutron spectra observed at $55^{\circ}$ (upper) $90^{\circ}$ (lower). The solid circles are the experimental results. The solid line is an ISNR fit with an anisotropic Maxwellian ion distribution model. The chi square per degree of freedom was 1.7. The dashed line is an ISNR fit with the adaptive ion spectrum model. The chi square per degree of freedom for this model was 1.1.

shots under the same conditions. The observed width of each spectrum $(\sim 1 \mathrm{MeV})$ was very much broader than that of a thermonuclear reaction in an implosion experiment (typically $\sim 100 \mathrm{keV}$ ). The observed emission was $7 \times 10^{4}$ neutrons per steradian for each detector.

\section{DISCUSSION}

\section{A. Confirmation of the neutron source}

In the high-intensity laser plasma experiment, excessive amounts of energetic electrons, photons, and ions are emitted from the interacting region. These energetic particles may cause a nuclear reaction on the material around the target and produce undesirable background neutrons [13]. For example, ${ }^{27} \mathrm{Al}(d, n)$ on instruments made of aluminum and $d(d, n)$ on a target positioner deuterated in the former experiment are suspected as nontarget neutron sources. In addition, $(\gamma, n)$ and $(e, n)$ reactions on instruments near the target are also possible. To confirm the neutron source, we covered almost $4 \pi$ sr of the target with a 15-mm-thick plastic shroud, excluding solid angles for laser irradiation and neutron observation. This shroud moderates the energetic ions and the electrons from the target. Since the insertion of the plastic shroud did not affect the neutron spectrum, we concluded that all the observed neutrons were produced in the target.

\section{B. Neutron production mechanism}

Since there was no neutron signal upon hydrate polystyrene $\left(\mathrm{C}_{8} \mathrm{H}_{8}\right)_{x}$ target irradiation, the neutrons were produced by deuteron-related reactions.
The distinctive difference between neutron spectra observed at $55^{\circ}$ and $90^{\circ}$ indicates that the neutron production mechanism is not conventional isotropic thermonuclear reactions. The thermonuclear fusion process can be described as follows. In hot plasma, deuterons collide due to their thermal motion. If the collision velocity (velocity of the relative motion of two ions) is sufficient, colliding two deuterons causes a nuclear reaction. In the laboratory system, the center of mass of the two reacting ions is also in thermal motion (i.e., parallel motion) with the Maxwellian velocity distribution. For $d(d, n)^{3} \mathrm{He}$ reactions, this parallel motion causes Doppler broadening of the neutrons. Since the velocity distribution of the parallel motion is isotropic, the neutron spectrum produced by thermonuclear reaction should be isotropic. Therefore, the thermal nuclear reaction is excluded from the possible neutron production mechanisms.

On the other hand, if energetic ions accelerated at the material surface react with nearly stationary ions in the material (i.e., a beam-target reaction), the relation of the energetic ion's anisotropy and the neutron's anisotropy is completely different from that of thermonuclear reactions. Since the energetic ion source is localized on the target surface, only the ions accelerated in the forward direction cause nuclear reactions and ions accelerated in the backward direction do not contribute to neutron production. Then even if the energetic deuteron production is isotropic, the velocity distribution of the center of mass of the two reacting ions is quite anisotropic (the center-of-mass velocity distribution spreads only in one hemisphere of the material in the velocity space). Therefore, an anisotropic neutron spectrum should be observed even if the deuteron acceleration is isotropic.

Table I shows the list of possible mechanisms of neutron production. $Q$ means the total energy release of each reaction. The mechanisms are (1) $d^{*}(d, n)^{3} \mathrm{He}$ : the energetic deuterons react with stationary deuterons; (2) $\mathrm{C}\left(d^{*}, n\right)^{13} \mathrm{~N}$ : the energetic deuterons react with stationary carbons; (3) $\mathrm{C}\left(d^{*}, n\right){ }^{13} \mathrm{~N}$ : the energetic carbons react with stationary deuterons; (4) $d\left(e^{*}, e^{\prime} n\right) p$ : electrodissociation of deuterons; and (5) $d\left(\gamma^{*}, n\right) p$ : photodissociation of deuterons. Particles marked with an asterisk mean energetic particles. Reactions (4) and (5) can be excluded, since the quantum efficiencies of neutron production with these mechanisms are small for a $5.5 \mu \mathrm{m}$ target $[16,17]$ and the required total energy of the energetic particles exceeds the input laser energy. From a point of energy conservation, neutron production due to charged-particle reactions (1), (2), and (3) are possible. However, since reactions (2) and (3) are endothermic (negative energy release), the energies of the neutrons produced by (2) and (3) should be lower than that produced by exothermic (positive energy release) reaction (1). Figure 3 shows the vector diagram of the charged-particle reaction. An incident particle (energetic deuteron or carbon) reacts with a target nucleus (stationary deuteron or carbon) in the target. Angles $\theta$ and $\theta^{\prime}$ are directions of a neutron in center-of-mass and laboratory systems. A laboratory energy $E_{3}$ of the neutron can be expressed as a function of the incident particle energy $E_{1}$ and $\theta$, 
TABLE I. Possible neutron production mechanisms. The species marked with an asterisk in the reaction field mean energetic particles. $E_{\text {inc }}$ is the particle energy required to produce $2.5 \mathrm{MeV}$ neutrons. $\eta$ represents the quantum efficiency of the neutron production by each reaction; this value is calculated from the density and thickness of the target and cross section of each reaction. The energy slowdown of the charged particles is also taken into account. $E_{\text {req }}$, which is the product of $E_{\text {inc }}$ and $\eta$, represents the required total energy of the energetic particles. Explanations (4) and (5) are excluded, since the energy $E_{\text {req }}$ cannot exceed the laser energy of $50 \mathrm{~J}$.

\begin{tabular}{|c|c|c|c|c|c|c|c|}
\hline Reaction No. & & $Q(\mathrm{MeV})$ & $E_{\text {inc }}$ & Cross section & $\eta$ & $E_{\text {req }}$ & Ref. \\
\hline (1) & $d^{*}(d, n)^{3} \mathrm{He}$ & 3.269 & $\sim 100 \mathrm{keV}$ & $\sim 40 \mathrm{mb}$ & $3 \times 10^{-8}$ & $\sim 500 \mathrm{~mJ}$ & [14] \\
\hline (2) & $C\left(d^{*}, n\right)^{13} \mathrm{~N}$ & -0.281 & $>3 \mathrm{MeV}$ & $\sim 200 \mathrm{mb}$ & $5 \times 10^{-6}$ & $\sim 100 \mathrm{~mJ}$ & [15] \\
\hline (3) & $C^{*}(d, n)^{13} \mathrm{~N}$ & -0.281 & $>8 \mathrm{MeV}$ & $\sim 150 \mathrm{mb}$ & $2 \times 10^{-6}$ & $\sim 640 \mathrm{~mJ}$ & [15] \\
\hline (4) & $d\left(e^{*}, e^{\prime} n\right) p$ & -2.225 & $\sim 5.2 \mathrm{MeV}$ & $\sim 10 \mu \mathrm{b}$ & $2.5 \times 10^{-10}$ & $\sim 3 \mathrm{~kJ}$ & [16] \\
\hline (5) & $d\left(\gamma^{*}, n\right) p$ & -2.225 & $\sim 5.2 \mathrm{MeV}$ & $\sim 2.5 \mathrm{mb}$ & $6 \times 10^{-10}$ & $\sim 90 \mathrm{~J}$ & [17] \\
\hline
\end{tabular}

$$
\begin{aligned}
E_{3}= & {\left[\frac{m_{1} m_{3}}{\left(m_{3}+m_{4}\right)^{2}}+\frac{m_{2} m_{4}}{\left(m_{1}+m_{2}\right)\left(m_{3}+m_{4}\right)}\right] E_{1}+\frac{m_{4}}{m_{4}+m_{3}} Q } \\
& +2\left[\frac{m_{1} m_{2} m_{3} m_{4}}{\left(m_{1}+m_{2}\right)\left(m_{3}+m_{4}\right)^{3}}\left(E_{1}^{2}+\frac{m_{1}+m_{2}}{m_{2}} Q E_{1}\right)\right]^{1 / 2} \\
& \times \cos \theta
\end{aligned}
$$

where $m_{1}, m_{2}, m_{3}$, and $m_{4}$ are the mass of the incident particle, target particle, neutron, and associate particle. Figure 4 shows the relation of the incident particle energy and the neutron energy described by Eq. (1) for each reaction. The gray-scale regions indicate the energy region of neutrons produced by each reaction. Upper and lower boundaries of each region correspond to forward and backward emission $\left(\theta=0^{\circ}\right.$ and $\left.180^{\circ}\right)$, respectively. Since reaction (1) $d^{*}(d, n)^{3} \mathrm{He}$ is exothermic, the energy of the neutron produced by this reaction spreads around $Q \times m_{4} /\left(m_{3}+m_{4}\right)$ $=2.45 \mathrm{MeV}$. The observed neutron spectrum shows good agreement with the energy region of this reaction. The neutrons produced by this reaction have a minimum energy of $1.63 \mathrm{MeV}$. This minimum can be explained as follows. The lowest-energy neutron is emitted backward in a center-ofmass system $(\cos \theta=-1)$. When $E_{1}<Q$, the negative influence of third term is dominant in Eq. (1). Then $E_{3}$ decreases with increase of $E_{1}$. However, for $E_{1}>Q$, the influence of the first positive term overcomes the third negative term and $E_{3}$ increases with $E_{1}$. Therefore, $E_{3}$ has a minimum at 1.63 $\mathrm{MeV}$ when $E_{1}=3.3 \mathrm{MeV}$. In contrast, (2) $\mathrm{C}\left(d^{*}, n\right){ }^{14} \mathrm{~N}$ and

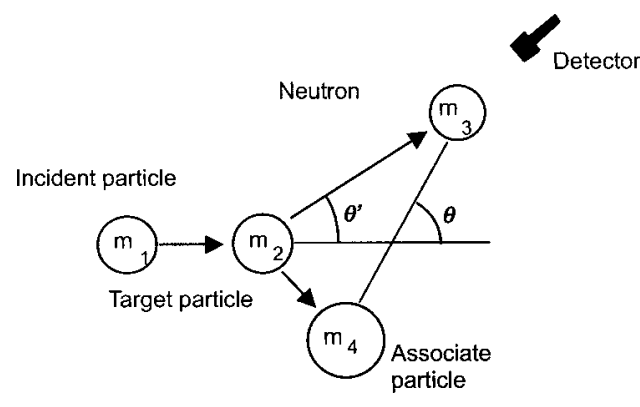

FIG. 3. A vector diagram of a nuclear reaction. An incident particle reacts with a stationary target particle. Angles $\theta^{\prime}$ and $\theta$ are the directions of a neutron in a laboratory system and a center-ofmass system, respectively.
(3) $\mathrm{C}^{*}(d, n){ }^{14} \mathrm{~N}$ are endothermic. The neutron produced by these reactions should be observed mostly below $1.6 \mathrm{MeV}$. We conclude that the observed broad peak around $2.5 \mathrm{MeV}$ is dominated by neutrons produced by $(1) d^{*}(d, n)^{3} \mathrm{He}$.

\section{Monte Carlo simulation of neutron production}

To study the directionality and the energy spectrum of the accelerated deuterons, we performed numerical experiments by using a three-dimensional (3D) Monte Carlo code named ISNR. This code calculates the spectra of neutrons produced by $d^{*}(d, n)^{3} \mathrm{He}$ reactions in a finite thickness target and emitted in specific directions of observation. In ISNR, energetic ions are started from a point on the target surface with a given velocity distribution. Initial velocities of energetic deuterons are given by appropriate simulated random numbers. The neutron flux to specific directions of observation during an infinitesimal path $\Delta x$ of the test particle is

$$
\Delta \frac{d N}{d \omega}=\frac{\rho_{d}}{m_{d}} \frac{d \sigma\left(E_{d}, \theta^{\prime}\right)}{d \Omega} \Delta x,
$$

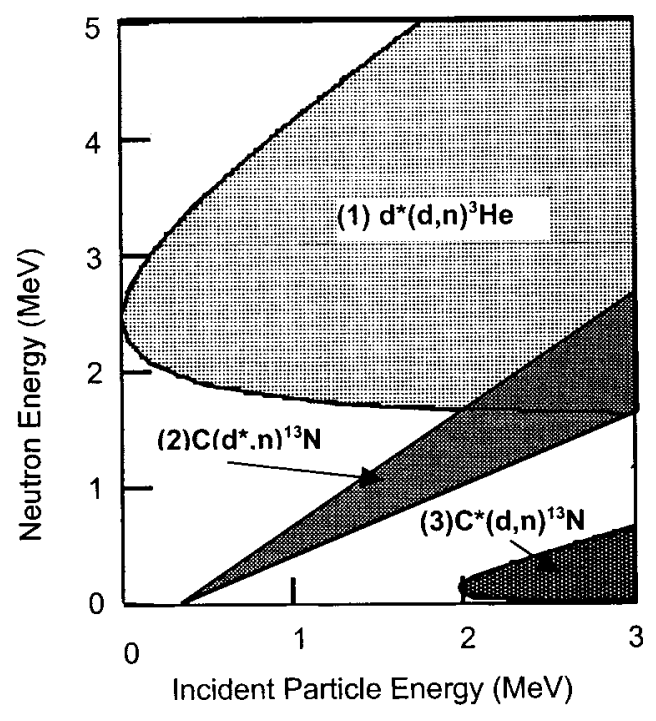

FIG. 4. The energy of neutrons produced by each nuclear reaction. The most energetic neutron is emitted forward $\theta=0^{\circ}$ and the least energetic is emitted backward $\theta=180^{\circ}$. The neutrons observed around $2.5 \mathrm{MeV}$ are produced predominantly by $d^{*}(d, n)^{3} \mathrm{He}$. 
where $\rho_{d}$ is the deuteron density in the target, $m_{d}$ is the deuteron mass, $E_{d}$ is the deuteron energy, $\theta$ is the neutron emission angle from the direction of incident deuteron in the laboratory system, and $d \sigma\left(E_{d}, \theta^{\prime}\right) / d \Omega$ is the differential cross section of the $d^{*}(d, n)^{3} \mathrm{He}$ reaction in the laboratory system. This value is calculated from the total cross section expressed by Bosh and Hale [18] and the angular distribution table in the center-of-mass system from ECPL86 [14]. The energy of the neutron produced in $\Delta x$ is given by

$$
\begin{aligned}
E_{n}= & \frac{m_{d} m_{n}}{\left(m_{n}+m^{3} \mathrm{He}\right)^{2}}\left\langle\cos \theta^{\prime} \pm\left\{\cos ^{2} \theta^{\prime}+\frac{m_{n}+m^{3} \mathrm{He}}{m_{d} m_{n}}\right.\right. \\
& \left.\left.\times\left[m_{{ }^{3} \mathrm{He}}\left(1+\frac{Q}{E_{d}}\right)-m_{d}\right]\right\}^{1 / 2}\right)^{2} E_{d},
\end{aligned}
$$

where $m_{n}, m^{3} \mathrm{He}$ are the mass of the neutron and ${ }^{3} \mathrm{He}$, respectively. The energy slowdown of the deuteron during $\Delta x$ is calculated as

$$
\Delta E=-\rho_{c d} \frac{d E\left(E_{d}\right)}{d \rho x} \Delta x,
$$

where $\rho_{c d}$ is the target density and $d E\left(E_{d}\right) / d \rho x$ is the stopping power of the deuteron. We used the charged-particle stopping power for cold material, which is calculated by SRIM96 [19]. Each test deuteron track is terminated when it reaches the rear surface or it decelerates below $1.5 \mathrm{keV}$. The neutron spectra produced by a substantial number of ions are integrated. (Typically, $10^{4}$ test particles were launched to represent an initial energy group of the energetic ions.)

\section{Anisotropy of the accelerated ions}

The angular distribution of the energetic ions is one of the important properties characterizing the acceleration mechanism. To investigate the anisotropy of the acceleration, we compared the observed neutron spectra and ISNR simulations with the given ion's angular distributions. Since the ion source is on the irradiated surface, the neutron productions are calculated only for one hemisphere of the material. As a working hypothesis, the velocity distribution of ions in numerical experiments is characterized by the anisotropic Maxwellian distribution

$$
f_{\text {ion }}=A \exp \left[-\frac{1}{2} m_{d}\left(\frac{v_{x}^{2}}{k_{B} T_{1}}+\frac{v_{y}^{2}+v_{z}^{2}}{k_{B} T_{2}}\right)\right],
$$

where $v_{x}, v_{y}$, and $v_{z}$ are the ion velocity component for each direction and $k_{B}$ is the Boltzmann constant. The target surface normal is on the $X$ axis. $T_{1}$ and $T_{2}$ are the temperatures of the longitudinal and the lateral directions. " $A$ " is an arbitrary constant. We sought the best pair of $T 1$ and $T 2$ that reproduces the observed neutron spectrum. As mentioned in the next section, the anisotropic Maxwellian model expressed by Eq. (5) may not be the best choice for fitting. However, the anisotropic Maxwellian model expressed by Eq. (5) is still useful for characterizing the ion's anisotropy. For a quantitative evaluation of the accuracy of the spectrum fitting, we used the chi-square evaluation. The chi-square per degree of freedom has a unique minimum at $T_{1}=90$ $\pm 10 \mathrm{keV}$ and $T_{2}=70 \pm 10 \mathrm{keV}$. The reduced chi square at this point is reasonably small, $\chi^{2} / \nu=1.7$. ( $\chi^{2}$ is a chi square that tests the accuracy of the fit and $\nu$ is a degree of freedom that is defined as the number of data points minus the number of parameters to be determined from the data points. The reduced chi square $\chi^{2} / \nu$ should be approximately equal to 1 [20].) The solid lines in Fig. 2 show the calculated neutron spectra, which minimize the chi square. The observed spectra (below $3 \mathrm{MeV}$ ) show good agreement with the numerical experiment. This result indicates that the deuteron acceleration was almost isotropic.

\section{E. Energy spectrum of accelerated ions}

The numerical experiments with the anisotropic Maxwellian model indicate that the ion acceleration was almost isotropic. However, the neutron spectra over $3.5 \mathrm{MeV}$ and the peak energy of the $55^{\circ}$ spectrum were not reproduced well with the anisotropic Maxwellian model. This indicates that the real spectrum has more high-energy and low-energy components compared to the anisotropic Maxwellian model. We calculated the ion spectrum that explains the observed neutron spectrum by using an unfolding calculation. In this analysis, we assumed that the ions were accelerated isotropically from a point on the target surface into a $2 \pi$ hemisphere of the target side. The ISNR code generates the neutron spectrum for a given ion energy spectrum of $30 \mathrm{keV}$ to $3 \mathrm{MeV}$. The ion spectrum is modified so that the neutron spectra of numerical experiment show the minimum chi square to the experimental spectra (adaptive ion spectrum model). The dashed lines of Fig. 2 show the neutron spectra of the numerical experiment, which minimize the chi square. The result of the adaptive ion spectra model reproduced the experimental neutron spectrum more neatly compared to the anisotropic Maxwellian model. The chi square per degree of freedom of this fitting is reasonably small $\left(\chi^{2} / \nu=1.11\right)$. Figure 5 shows the ion spectrum obtained by chi-square minimization. Each error bar shows the independent confidence interval of each parameter. To find the confidence intervals, one parameter was deviated so that the chi square increased by 1 from the minimum $\left(\Delta \chi^{2}=\chi^{2}-\chi_{\min }^{2}=1\right)$. During this trial, other parameters were allowed to seek their optimum values [20]. The confidence level inside the confidence interval is $68.3 \%$. As expected qualitatively from previous discussion, the ion spectrum obtained with the adaptive ion spectrum model has more low- and high-energy components compared to the anisotropic Maxwellian model. The deuteron spectrum from $30 \mathrm{keV}$ to $3 \mathrm{MeV}$ scales the negative third power of deuteron energy.

\section{F. Energy transport}

The energy transfer from laser to ions is crucial for quantitative understanding of the interaction dynamics. The calculated total energy of the deuterons from $30 \mathrm{keV}$ up to 3 $\mathrm{MeV}$ was $0.9 \mathrm{~J}$ ( $\sim 2 \%$ of the laser energy). The estimation of the energy transfer to the energetic carbons is uncertain, since the ionizing charge states of the energetic carbons were not observed. Assuming the full stripping of the carbons in 


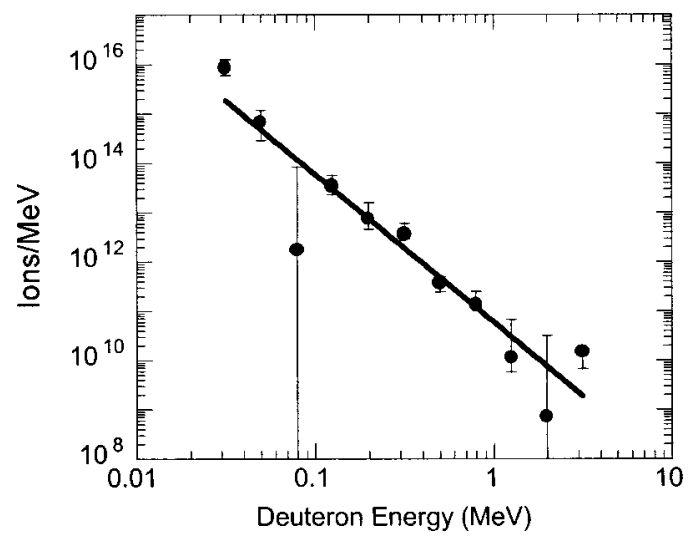

FIG. 5. The deuteron spectrum that gives the minimum chisquare fitting to the observed neutron spectrum by the Monte Carlo calculation. Each error bar shows an independent confidence interval of $68.3 \%$ confidence level. The deuteron spectrum scales along with negative third power of the deuteron energy. The solid line shows a fitting with $P\left(E_{d}\right) \sim E_{d}{ }^{-3}$.

the interaction region, the charge-to-mass ratio of the accelerated carbons is the same as the deuterons $(Z / A \sim 0.5)$. Therefore, the carbons could be accelerated to the same velocities as the deuterons. Since the ratio of the carbons to the deuterons in polystyrene is almost unity, the number of the accelerated deuterons and carbons could be the same. Under these assumptions, the energy flow to the carbons could be six times as high as that to deuterons. The energy transferred to all ions could be up to $6.3 \mathrm{~J}$ (13\% of the laser pulse energy).

\section{G. Ion acceleration mechanism}

The mechanism of high-energy ion production from ultraintense laser irradiation is a matter of interest. Due to the difference in the particle mass, the energy coupling of laser to electrons is much stronger than ions. First the laser heats the electrons. Then the thermal energy of the electron is to the ions. The unique point of the ultrashort pulse laser interaction is that the pulse can heat up the electrons to extremely high temperatures before the ions are heated and the initial density profile is modified. Then a strong electrostatic field is formed on a steep boundary of the target surface and the ions are accelerated coherently by the field.

In this experiment, there was a prepulse at $800 \mathrm{ps}$ prior to the main pulse with $10^{-4}$ of the main pulse energy. Therefore, there must have been preformed plasma with a scale length of a few tens of micrometers and the main pulse interacts with it. In this situation, the ion acceleration on an irradiated surface is well modeled by Wilks et al. as a holeboring model [21]. The hole-boring model was described in two steps: (1) formation of an electrostatic potential at the laser plasma interface and (2) sweeping of the ions by the propagating electrostatic potential.

Since the electrons on the irradiated surface are heated immediately while the ions are still cold, the electrons explode until the Coulomb attractive force between the ions and the electrons balances the thermal pressure of the electrons. The local violation of the charge neutrality causes a

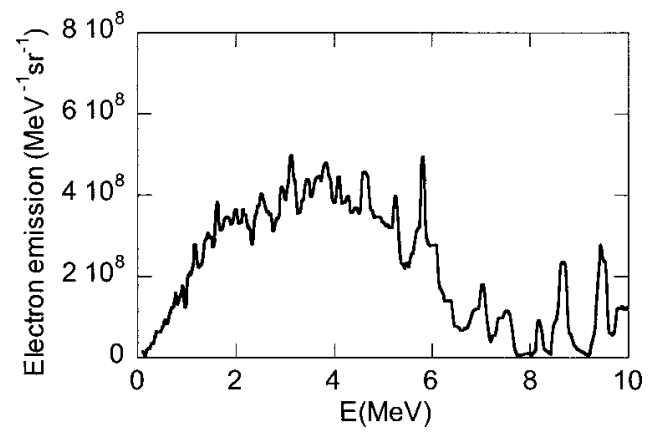

FIG. 6. The electron spectrum observed at $42^{\circ}$ from the laser propagation direction. The observed electrons had a broad peak around $4 \mathrm{MeV}$.

strong charge separation layer at the laser/plasma interface. The electrostatic potential of the charge separation layer gives the highest energy of the accelerated ions. The electrostatic potential $\phi$ is sustained by electron temperature. One can roughly estimate the electron temperature $T_{e}$ by using heuristic scaling of the ponderomotive potential. Therefore, the electrostatic potential $\phi$ can be estimated by

$$
\phi \approx T_{e} \approx m_{e} c^{2}\left[\left(1+\frac{I \lambda^{2}}{1.37 \times 10^{18}}\right)^{1 / 2}-1\right],
$$

where $m_{e}$ is the electron mass, $c$ is the light velocity, $I$ is the irradiation intensity in $\mathrm{W} \mathrm{cm}^{-2}$, and $\lambda$ is the laser wavelength in micrometers. For this case, $I \lambda^{2}=2 \times 10^{19} \mathrm{~W} \mu \mathrm{m}^{2} \mathrm{~cm}^{-2}$, the electrostatic potential $\phi$ could be $1.5 \mathrm{MeV}$. In the experiment, the electron energy spectrum was also observed simultaneously. Figure 6 shows the electron spectrum observed at an angle of $42^{\circ}$ to the laser propagation direction. The spectrum had a broad peak around $4 \mathrm{MeV}$. The spectrum was more energetic than the estimation of Eq. (6), but qualitatively consistent.

The radiation pressure of focal spot $P_{L} \approx(1 \sim 2) I / c$ is much higher than the thermal pressure of the preformed plasma. In this case the radiation pressure could be as high as 6.5-13 Gbar. Then the laser-plasma interface is pushed into the material together with the strong electrostatic potential. The ions, which are in the upstream and cannot penetrate the potential $\phi$, are swept into the forward part of the potential. The velocity of the laser-plasma interface can be estimated from conservation of momentum and mass [21],

$$
\frac{u}{c}=\left(\frac{n_{c}}{2 n_{p e}} \frac{Z m_{e}}{M} \frac{I \lambda^{2}}{1.37 \times 10^{18}}\right)^{1 / 2},
$$

where $n_{c}$ is the critical density of the laser pulse and $n_{p e}$ is the electron density of the recessing interface, $M$ is the ion mass, and $Z$ is the charge state of the ions. Assuming that the interface sweeps the preformed plasma at the region from $n_{p e}=n_{c}$ to $4 n_{c}$, the velocity of the deuteron swept by the front could be $u=0.045 c$ to $0.022 c$. These velocities correspond to the deuteron energy of $2 \mathrm{MeV}$ and $500 \mathrm{keV}$. These estimations are consistent with the kinetic energy of the deuterons calculated from the neutron spectra (Fig. 5).

When the target thickness is thinner than the stopping range of the energetic electrons, hot electrons can penetrate 
through the target and cause strong charge separation also on the rear surface. Efficient ion acceleration from the rear surface into vacuum by this charge separation is also reported [22]. Since we observed the nuclear reactions inside the dense target, our experiment is insensitive to the outward ions from the rear surface. Therefore, the front surface acceleration is clearly separated from that on the rear surface.

Here we would like to discuss why the observed deuteron distribution was almost isotropic. It is interesting to compare our result with the collimated ion acceleration reported by Disdier et al. [8] and Disdier, Garçonnet, and Miquel [9]. In Ref. [8], they irradiated the deuterated plastic target $\left(\mathrm{CD}_{2}\right)$ using an ultraintense laser pulse (pulse duration $\tau<300 \mathrm{fs}$, focal spot diameter $\phi=5 \mu \mathrm{m}$, wavelength $\lambda=529 \mathrm{~nm}$, peak intensity $I=2 \times 10^{19}$ to $\left.3 \times 10^{19} \mathrm{~W} / \mathrm{cm}^{2}\right)$. The observed neutron yield was up to $10^{6}\left(8 \times 10^{4}\right.$ neutrons per steradian $)$ when $I=2 \times 10^{19} \mathrm{~W} / \mathrm{cm}^{2}$. The neutron yield was almost identical to ours. However, they observed distinctively different angular distribution of neutrons. The intensity of the neutrons observed from $0^{\circ}$ was about three times larger than that for $100^{\circ}$. This indicates strongly collimated ion acceleration into the target normal. We suppose that the most important difference between these experiments was the contrast ratio of prepulse to main pulse. Their contrast ratio was $10^{12}$ and the irradiation intensity before the main pulse was insufficient to ionize the target, so the laser pulse interacts directly with the solid target and not with a preformed plasma. Since the electrostatic field of the laser-plasma interface sweeps the ions, the shape of the interface determines the directionality of the ions. When the charge separation of the interface is planar, the ions kicked by the interface are collimated. However, when the interface is bowed, the ions will spray into a large solid angle. The aspect of the interface bowing is determined by the focal spot size and the recession depth. Since the laser intensity is higher at the spot center, the interface recession is faster at the center and then the interface is bent. In our experiment, there might have been a preformed plasma with a scale length of a few tens of micrometers. The recession depth during the laser pulse duration could be comparable to the diameter of a focal spot $\sim 5.4 \mu \mathrm{m}$. Therefore, it is reasonable to assume significant bowing of the interface. In contrast, the density scale length of the laser plasma interface in Ref. [8] was very steep and the recession depth might have been very shallow. Then the shape of the charge separation was almost planar and the accelerated ion was collimated.

\section{H. Comparison with particle-in-cell simulations}

The relation of interface bowing to ion diversion is confirmed with particle-in-cell (PIC) simulations. The ion acceleration was compared for steep (case $S$ ) and a moderate (case $L$ ) initial boundary conditions. The size of the simulation box was $X=40 \mu \mathrm{m} \times Y=10 \mu \mathrm{m}$. The target was irradiated from the left side $(X=0)$. Fully ionized plasma was assumed as an initial target condition. The charge-to-mass ratio of the ions $Z / A=0.5$. This is equivalent to the fully ionized deuteron and carbon plasma. The target density was $40 n_{c}(40$ times the critical density). The target thickness was 5 and 3 $\mu \mathrm{m}$ for cases $S$ and $L$, respectively. Therefore, the irradiated surface position was $X=10(12) \mu \mathrm{m}$ in the case $S(L)$. Each target had a density-ramped preformed plasma on the irradiated surface. Their density slopes from 0 to $15 n_{c}$. Their scale lengths were $1 \mu \mathrm{m}$ for case $S$ and $7 \mu \mathrm{m}$ for case $L$, respectively. In our experiments, there was a prepulse at 0.8 ns prior to the main pulse and the contrast ratio was $10^{4}$. Assuming that a few percent of the prepulse energy was absorbed in the skin depth, the estimated expansion of preformed plasma is a few tenths of a micrometer. Therefore, the initial condition of case $L$ is much closer to our experiment. The dimensionless laser amplitude $a$ is defined as $a$ $=e \mathbf{E} /\left(m_{e} c \omega_{0}\right)$, where $e$ is the electron charge magnitude, $\mathbf{E}$ is an electric field of the laser irradiation, $m_{e}$ is the electron rest mass, $c$ is the speed of light, and $\omega_{0}$ is the angular velocity of the laser field. In each case, the intensity of the laser irradiation $a$ is equal to 3.3 and it corresponds to 2 $\times 10^{19} \mathrm{~W} / \mathrm{cm}^{2}$. The intensity profile of the focal spot is the Gaussian with $5 \mu \mathrm{m}$ full width at half maximum. The laser pulse is irradiated from the boundary $X=0$ with $P$ polarization (electric field vector is in the $X-Y$ plane). The pulse rises by $50 \mathrm{fs}$ with the Gaussian temporal profile, keeps the peak intensity for $300 \mathrm{fs}$, and then terminates it in $50 \mathrm{fs}$. The total time duration of the simulation is 160 laser periods (530 fs). The simulations are performed with $4000 \times 1024$ meshes and $53 \times 10^{6}$ particles.

Figure 7 shows the ion density profile of each case. Several "bowlike" collisionless shock fronts could be seen in case $L$ [Figs. 7(I),7(II)]. In contrast, the irradiated surface of case $S$ keeps flat shape. Since the initial boundary is steeper, the recession depth of case $S$ is shallower than the spot size [Fig. 7(III)]. In case $L$, the laser pulse is stopped around $X$ $=7 \mu \mathrm{m}$ where the plasma density corresponds to the relativistic critical density $\gamma_{o s} n_{c}$; here $\gamma_{o s}$ is given by $\left(1+a^{2}\right)^{0.5}$ $\sim 3.5$. Then the laser pulse starts boring the plasma with the electrostatic fields of the charge separation caused by its photon pressure. We found that these electrostatic fields have time-oscillating components with two fundamental frequencies: high and low. The high frequency is the $2 \omega_{0}$ due to the $\mathbf{J} \times \mathbf{B}$ force; here $\omega_{0}$ is the laser frequency. The low frequency is close to the ion acoustic frequency at the relativistic critical density; $\omega_{\mathrm{pi}}=\left(\gamma_{\mathrm{os}} m_{e} / M\right)^{0.5} \omega_{0} \sim 0.03 \omega_{0}$. The high-frequency fields push the electrons twice in a laser oscillation period and generate fast electron trains mainly in the forward direction $[21,23]$. In contrast, ions are practically insensitive to the high-frequency component. Since the deuteron is 3600 times heavier than electron, the momentum transfer to deuterons in a short oscillation period is not significant. Therefore, only the low-frequency component affects the ion's motion and it enhances the ion acceleration intermittently, every $2 \pi / 0.03 \omega_{0} \sim 100 \mathrm{fs}$. In consequence, multiple collisionless shocks are formed. In Fig. 7, we can see two collisionless shocks at 264 fs [Fig. 7(I)] and three shocks at 396 fs [Fig. 7(II)].

To show the details of the ion acceleration mechanism, we made an $X$ - $t$ diagram of the ion density profile at the center ( $Y=5 \mu \mathrm{m}$ ) (Fig. 8). The color indicates the ion density. The slopes of the density contour give the propagation velocities of the laser-plasma interface and the shock fronts. The laser- 

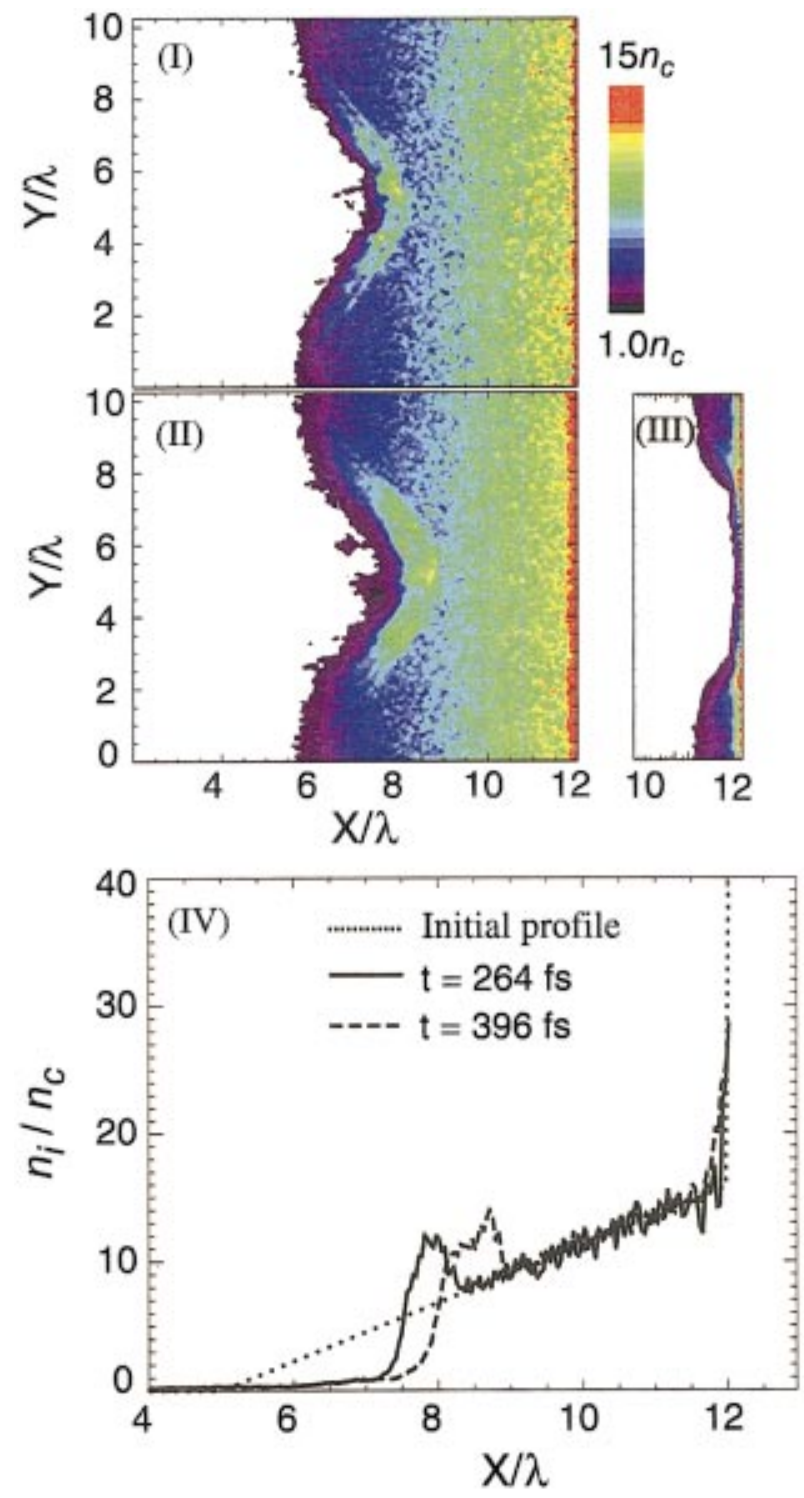

FIG. 7. (Color) (2D-PIC) The ion density profile of case $L$, observed at 264 fs (I) and 396 fs (II). (III) shows the ion density profile in the case $S$ at 200 fs. (IV) is the slice of (I) and (II) at $Y$ $=5 \mu \mathrm{m}$. The dotted line indicates the initial density profile of case $L$.

plasma interface velocity is well explained by the holeboring model with Eq. (7). The kinetic energy of deuterons, which is swept by the interface propagating with the velocity $u$, can be calculated by $E_{d}=\left(m_{d} u^{2}\right) / 2$, where $m_{d}$ is the deuteron mass. When the interface propagates through the underdense region (density below the relativistic critical density $\gamma_{\mathrm{ps}} n_{c}$ ), the surface speed $u$ is about $0.04 c$ (it corresponds to $\left.E_{d}=1.44 \mathrm{MeV}\right)$. Then, when the interface passes through the point of $\gamma_{o s} n_{c}$, the interface decelerates to $0.017 c\left(E_{d}\right.$ $=278 \mathrm{keV})$ and finally to $0.011 \mathrm{c}\left(E_{d}=110 \mathrm{keV}\right)$. The ion phase $\left(X-P_{x}\right)$ plot of Fig. 9 gives an overview of ion acceleration. Figures 9(a) and 9(b) show the result of cases $S$ and $L$, respectively. The target is irradiated from the left side. Through the simulations, the absorption rate of the input laser is $42.8 \%$ in case $S$ and $60.3 \%$ in case $L$. The higher laser

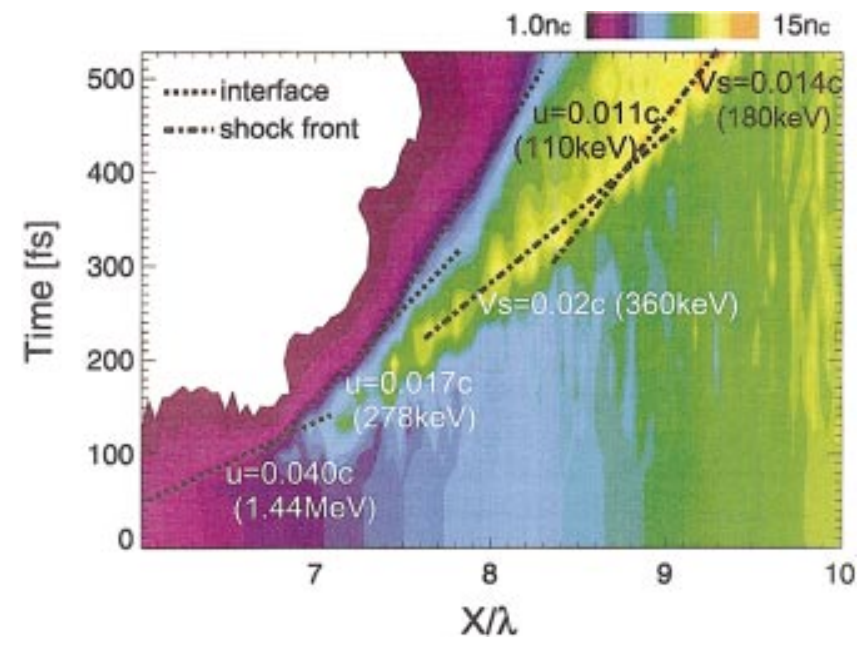

FIG. 8. (Color) (2D-PIC) The $X$ - $t$ diagram of the ion density at $Y=5 \mu \mathrm{m}$ in case $L$.

absorption and efficient generation of energetic electrons are observed in case $L$. As shown in Fig. 9, the ion acceleration occurs not only at the irradiated surface but also at the rear surface. At the irradiated surface, the maximum energy of ions in case $L(2 \mathrm{MeV})$ is greater than that of case $S(1$ $\mathrm{MeV})$. This relation is well explained by the hole-boring model by substituting different plasma densities for Eq. (7).

When the energetic electrons, which are generated on the irradiated surface, pass through the target, they excite a strong electrostatic field at the rear surface. Therefore, strong ion acceleration also takes place at the rear surface [24]. The maximum energy of the ions accelerated at the rear surface is greater than that of the ions accelerated at the irradiated sur-

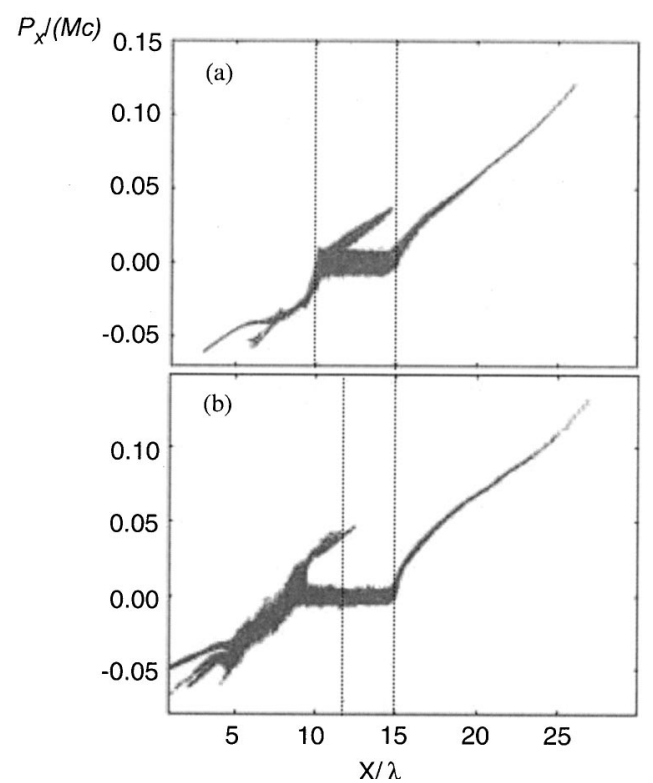

FIG. 9. (2D-PIC) The ion phase $\left(X-P_{x}\right)$ plot at 598 fs. Figure 9 (a) is for case $S$, (b) is for case $L$. The dotted lines indicate the initial surface of the solid target. The target is irradiated by laser pulse from the left side. The space $X$ is normalized by the laser wavelength $\lambda$, the ion longitudinal momentum is normalized by $M \times c$. 


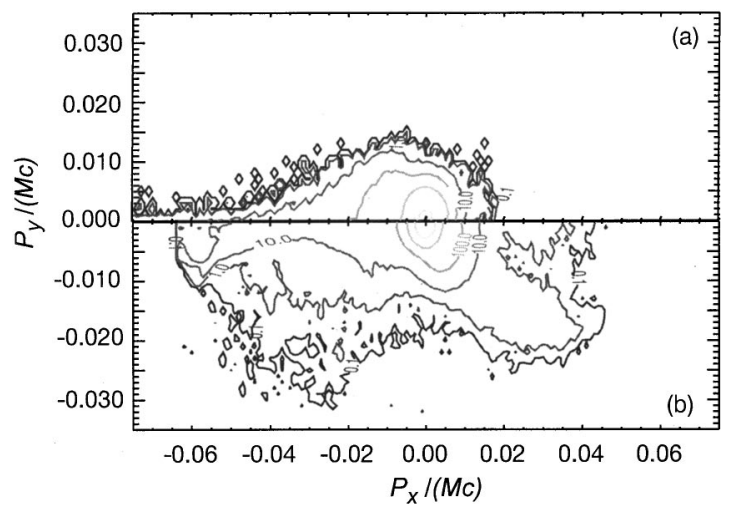

FIG. 10. (2D-PIC) The angular distribution of the accelerated ions in a momentum space. The propagation of laser light is from left to right. The momentum is normalized by $M \times c$. These plots were observed at $530 \mathrm{fs}$. The upper (lower) plot is the result of case $S(L)$.

face. Since the laser absorption at the irradiated surface is higher for case $L$, the maximum energy of the ions accelerated at the rear surface is greater for case $L(19 \mathrm{MeV})$ than for case $S(13 \mathrm{MeV})$.

Figure 10 shows the PIC result of the ion momentum distribution in cases $S$ (a) and $L$ (b). Since the energetic deuterons react with deuterons in the target, the observed neutron spectra reflect the distribution of ions accelerated into the target. The rear side acceleration emits the ions outward and contributes less to the neutron production. To compare with the experiment, only the ions from the front surface are sampled in the simulation. There are several differences between the two cases. In case $L$, the ions are more energetic and have spread directionality, especially in the high-energy region $(|P|>0.01 ;>180 \mathrm{keV})$. In contrast, the ions are less energetic and more collimated into the $P_{x}$ axis in case $S$. As discussed in the preceding section, the observed neutron spectrum indicates the almost isotropic acceleration of the deuterons. This "spread" acceleration is well explained by an interface bowing that is distinctive in the case with a moderate density profile preformed plasma such as case $L$.

Figure 11 shows the ion spectra obtained by PIC simulations (multiplied by 70 to fit the experimental result). To compare with the experiment, the ions accelerated into the target are sampled. The ion spectrum calculated from the observed neutron spectrum is also plotted with the PIC results. As shown in Fig. 11, the ion energy spectrum of case $L$ has a transition around $200 \mathrm{keV}$. The ions above this energy are accelerated by the collisionless shocks that are generated at the early time stage ( $\sim 150 \mathrm{fs}$, see Fig. 8$)$. The velocities of the collisionless shock fronts are greater than that of the laser-plasma interface. The ions below $200 \mathrm{keV}$ are dominated by the ions kicked by the laser-plasma interface. The ion velocities in this region correspond to the laser-plasma interface velocity.

The influence of the preformed plasma on the ion's directionality was explained qualitatively. However, the yield of the energetic ions in the PIC simulation still has unexplained disagreement with the experimental result. Assuming that the

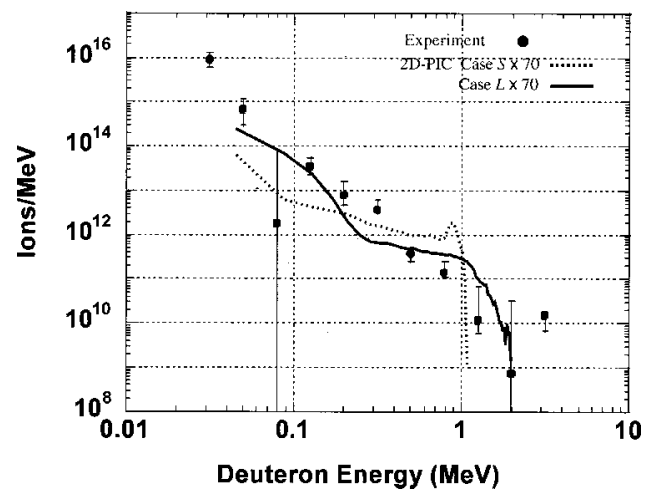

FIG. 11. (2D-PIC) The energy distribution of the ions accelerated into the target. The solid line is the result for case $L$ and the dotted line is that for case $S$ at 530 fs. The simulation results are multiplied by 70 to fit the experimental results.

extent of the hidden direction of the two-dimensional simulation (perpendicular to the simulation plane) is the same as the laser spot size, the energetic ion yield of the PIC simulation is 70 times smaller than that is calculated from the neutron spectrum. We suppose that the following effects might cause this discrepancy.

(1) The ionization states of the carbons affect the acceleration efficiency of the deuterons. In the PIC simulations, full ionization of the carbons is assumed in initial target conditions. Since the $Z / A$ of the deuterons and the fully ionized carbons are the same, the acceleration energy per nucleon must be the same for the deuterons and the carbons. Thus the energy flow to the deuterons is decided by the mass ratio of the deuterons and the carbons in the target plasma. Since the mass ratio of the deuterons and the carbons is $1: 6$, we expected that $1 / 7$ of the ion's total energy would be given to the deuterons. However, if the carbons were not ionized fully, the charge-to-mass ratio $Z / A$ of the carbons would be lower and the acceleration of the carbons less efficient than those of the deuterons. In this case, the energy flow to the deuterons could be higher than that of the simulation results with the full ionization model. This effect can increase the deuteron acceleration efficiency of the simulation up to a factor of 7.

(2) The dimensional effects: In two-dimensional simulations, the laser-plasma interface has the shape of a "blunt wedge." When the blunt wedge drives into the target, the wedge front rakes ions and the ion density of the upstream is easily piled up. Then interface velocity is decelerated [Eq. (7)]. In the three-dimensional case, the interface has the shape of a blunt nail and the upstream ions can be swept out in the radial direction. Thus fewer ions pile up in the upstream of the front. Then the driving velocity and depth could be faster and deeper for the three-dimensional case. Therefore, the number of ions in the volume swept by laser/ plasma interface is smaller for the two-dimensional case and the ion acceleration in the two-dimensional simulation is less efficient than the experiment. This effect will be confirmed by a $3 \mathrm{D}$ simulation code in the near future.

(3) The stopping power effects: In the numerical experiment with the ISNR, the stopping power for the cold solid material was used and it might have overestimated the 
charged-particle stopping in the target. If the target is ionized before the ions pass through, the ion stopping power in the target might be smaller than in the cold material. So the ion production calculated from the neutron spectrum might be overestimated.

For a more precise understanding of the ion acceleration on the irradiated surface, these uncertain factors should be investigated in future work. Recent intensive work by Toupin, Lefebvre, and Bonnaud with PIC calculations and the postprocess package for ion transport and neutron production shows qualitative agreement with our results [25].

\section{CONCLUSION}

We have observed $7 \times 10^{4}$ per steradian of the neutrons produced in a deuterated polystyrene target [material $\left(\mathrm{C}_{8} \mathrm{D}_{8}\right)_{x}$, thickness $\left.5.5 \mu \mathrm{m}\right]$ irradiated by a 500 -fs laser focused in a diameter of $5.4 \mu \mathrm{m}$ with an intensity of $2 \times 10^{19} \mathrm{~W} / \mathrm{cm}^{2}$. The fast neutron spectra were measured with multichannel time-of-flight neutron spectrometers at $55^{\circ}$ and $90^{\circ}$ to the rear target normal. The mechanism of neutron production is explained by nuclear reactions caused by the energetic deuterons accelerated at the irradiated sur- face. The directionality of the energetic ions was calculated by numerical experiments with a Monte Carlo simulation code. The energy spectrum of the energetic ions scaled negative third power of the ion's energy. The observed energy shows good agreement with the estimation by the holeboring model. The directionality was almost isotropic. To understand the mechanism of the spread ion acceleration, we performed PIC simulations. The ion acceleration on irradiated surfaces was well explained by the hole-boring model and collisionless shock acceleration. The spread ion directionality observed in the experiment is explained by bowing of the laser plasma interface, which is distinctive with moderate profile preformed plasma in front of the target.

\section{ACKNOWLEDGMENTS}

We are grateful to the members of the Laser, Target, and Measurement Tech. for their technical assistance. N.I. acknowledges S.C. Wilks for useful discussions. Y.S. acknowledges the ILE Computational staff for their support. The PIC simulations were performed on NEC SX5 at Cyber Media Center, Osaka University.
[1] P. Maine et al., IEEE J. Quantum Electron. 24, 398 (1988); M. Ferray et al., Opt. Commun. 75, 278 (1990); M. D. Perry et al., Opt. Lett. 15, 381 (1990); J. P. Watteau et al., Phys. Fluids B 4, 2217 (1992).

[2] D. Umstadter et al., Science 273, 472 (1996); A. Modena et al., Nature (London) 377, 606 (1995); G. Malka et al., Phys. Rev. Lett. 79, 2053 (1997); K. B. Wharton et al., ibid. 81, 822 (1998); T. E. Cowan et al., ibid. 84, 903 (2000).

[3] M. Tabak et al., Phys. Plasmas 1, 1626 (1994).

[4] A. P. Fews et al., Phys. Rev. Lett. 73, 1801 (1994).

[5] A. R. Bell et al., Phys. Rev. E 48, 2087 (1993); A. P. Fews et al., Phys. Rev. Lett. 73, 1801 (1994); F. N. Beg et al., Phys. Plasmas 4, 447 (1997); E. L. Clark et al., Phys. Rev. Lett. 84, 670 (2000); A. Maksimchuk et al., ibid. 84, 4108 (2000); E. L. Clark et al., ibid. 85, 1654 (2000); R. A. Snavely et al., ibid. 85, 2945 (2000).

[6] G. Pretzler et al., Phys. Rev. E 58, 1165 (1998).

[7] P. A. Norreys et al., Plasma Phys. Controlled Fusion 40, 175 (1998); M. H. Key et al., Phys. Plasmas 5, 1966 (1998).

[8] L. Disdier et al., Phys. Rev. Lett. 82, 1454 (1999).

[9] L. Disdier, J.-P. Garçonnet, and J.-L. Miquel, in Inertial Fusion Sciences and Applications 99, edited by C. Labaune, W. J. Hogan, and K. A. Tanaka (Elsevier, Paris, 2000), pp. 10261031.

[10] K. A. Tanaka et al., Phys. Plasmas 7, 2014 (2000); For oblique irradiation experiment, H. Habara et al., Proc. SPIE 3886, 513 (2000).

[11] H. Fujita et al., Proc. SPIE 3047, 501 (1997).

[12] N. Izumi et al., Rev. Sci. Instrum. 70, 1221 (1999).

[13] For work about nontarget neutron production, see P. A. Norreys et al., Plasma Phys. Controlled Fusion 40, 175 (1998); T.
E. Cowan et al., Phys. Rev. Lett. 84, 903 (2000); R. A. Snavely et al., ibid. 85, 2945 (2000); for work about neutrons produced by $(n, \gamma)$ reaction, see T. W. Phillips et al., Rev. Sci. Instrum. 70, 1213 (1999).

[14] R. J. Howerton et al., Index to the LLNL Evaluated ChargedParticle Library, Vol. 28 (OECD Nuclear Energy Agency, Moulineaux, France, 1986).

[15] R. J. Jaszczak et al., Phys. Rev. 181, 1428 (1969).

[16] D. Harder et al., Phys. Lett. 32B, 610 (1970).

[17] E. Hadjimichael et al., Phys. Rev. C 36, 44 (1987).

[18] S.-H. Bosh and G. M. Hale, Nucl. Fusion 32, 611 (1992).

[19] J. F. Ziegler, J. P. Biersack, and U. Littmark, The Stopping and Range of Ions in Solids (Pergamon, New York, 1996).

[20] Philip R. Bevington and D. Keith Robinson, Data Reduction and Error Analysis for the Physical Sciences, 2nd ed. (McGraw-Hill, New York, 1992), pp. 194-195. For determination of the confidence region of Fig. 5, see also pp. 212214.

[21] S. C. Wilks et al., Phys. Rev. Lett. 69, 1383 (1992); S. C. Wilks, Phys. Fluids B 5, 2603 (1993); W. L. Kruer and K. Estabrook, Phys. Fluids 28, 430 (1985).

[22] Stephen P. Hatchett et al., Phys. Plasmas 7, 2076 (2000); R. A. Snavely et al., Phys. Rev. Lett. 85, 2945 (2000); A. J. Mackinnon et al., Phys. Rev. Lett. 86, 1769 (2001).

[23] Y. Sentoku, K. Mima, T. Taguchi, S. Miyamoto, and Y. Kishimoto, Phys. Plasmas 5, 4366 (1998).

[24] S. C. Wilks, A. B. Langdon, T. E. Cowan, M. Roth, M. Singh, S. Hatchett, M. H. Key, D. Pennington, A. MacKinnon, and R. A. Snavely, Phys. Plasmas 8, 542 (2001).

[25] C. Toupin, E. Lefebvre, and G. Bonnaud, Phys. Plasmas 8, 1011 (2001). 